\title{
Influences on torque measurement in nacelle test benches and their effect on the measurement uncertainty and consequences of a torque calibration
}

\author{
Gisa Foyer ${ }^{1}$, Stefan Kock², Paula Weidinger ${ }^{1}$ \\ ${ }^{1}$ Physikalisch-Technische Bundesanstalt, Bundesallee 100, 38116 Braunschweig, Germany \\ ${ }^{2}$ Chair for Wind Power Drives, Campus-Boulevard 61, 52062 Aachen, Germany
}

\begin{abstract}
Torque measurement in the $\mathrm{MN} \cdot \mathrm{m}$ range is important in nacelle test benches, as it constitutes the primary value for the efficiency determination of wind turbine drive trains. However, no traceable measurement is possible above $1.1 \mathrm{MN} \cdot \mathrm{m}$. At the moment, alternatives to direct torque measurement in the $\mathrm{MN} \cdot \mathrm{m}$ range are, therefore, used. Several of these torque measurement methods are presented and discussed in this paper with respect to traceability and measurement uncertainty. It was found that these methods cannot fully substitute direct measurements. Therefore, detailed recommendations for a torque calibration procedure based on the specific conditions in nacelle test benches (such as rotation and additional mechanical loads) are given so as to enable the traceability of torque measurement using a torque transfer standard.
\end{abstract}

\section{Section: RESEARCH PAPER}

Keywords: torque measurement; measurement uncertainty; nacelle test bench calibration; wind energy

Citation: Gisa Foyer, Stefan Kock, Paula Weidinger, Influences on torque measurement in nacelle test benches and their effect on the measurement uncertainty and consequences of a torque calibration, Acta IMEKO, vol. 8, no. 3, article 10, September 2019, identifier: IMEKO-ACTA-03 (2019)-01-10

Editor: Maija Ojanen-Saloranta, VTT Technical Research Centre of Finland, National Metrology Institute MIKES, Finland

Received August 23, 2018; In final form July 1, 2019; Published September 2019

Copyright: This is an open-access article distributed under the terms of the Creative Commons Attribution 3.0 License, which permits unrestricted use, distribution, and reproduction in any medium, provided the original author and source are credited.

Funding: This work has been funded by the EMPIR initiative which is co-funded by the European Union's Horizon 2020 research and innovation programme and the EMPIR Participating States.

Corresponding author: Gisa Foyer, e-mail: gisa.foyer@ptb.de

\section{INTRODUCTION}

Wind energy is a developing market initiated by a worldwide move towards renewable energies. This is leading to more as well as larger wind turbines, which must be tested prior to their market launch. Several nacelle test benches (NTBs) have been constructed worldwide in the last few years. These NTBs aim to accelerate the testing phase of innovative concepts and enable an overall system test compared to component testing.

One example of an NTB is shown in Figure 1. It consists of a prime mover, a load application system (LAS), and a device under test (DUT). The prime mover in Figure 1 is an electrical direct drive; other NTBs have a geared drive that reduces the speed and increases the torque between the electrical drive and the DUT. The LAS shown in Figure 1 is not found in all NTBs. Its purpose is the generation of additional load components (axial and lateral forces as well as bending moments) to the main shaft in order to simulate the mechanical loads acting on the rotor hub of a nacelle during field operation. A DUT, which is normally a nacelle but can also be a gearbox or a main bearing, is connected to the main shaft of the NTB for testing.

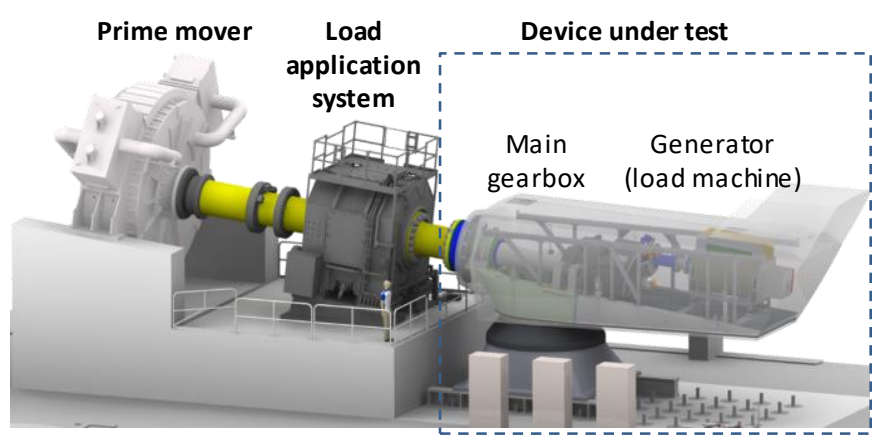

Figure 1. Example of a nacelle test bench: CWD Aachen, $4 \mathrm{MW}$ system test bench (Source: CWD Aachen) 
The tests on NTBs generally include torque measurements to determine the input power to the DUT. Depending on the purpose of the measurements, a measurement uncertainty of $0.1 \%$ for the torque measurement is envisioned [1]. However, the nominal torque load on most NTBs is in the multi-MN.m range, e.g. $13 \mathrm{MN} \cdot \mathrm{m}$ at the Fraunhofer Institute for Wind Energy and Energy System Technology (IWES), $6 \mathrm{MN} \cdot \mathrm{m}$ at the Centro National de Energías Renovables (CENER), and $3.4 \mathrm{MN} \cdot \mathrm{m}$ at the Center for Wind Power Drives of RWTH Aachen University, whereas the largest torque standard is only able to exert torque loads up to $1.1 \mathrm{MN} \cdot \mathrm{m}$ [2]. Moreover, the input torque to the DUT should ideally be measured directly at the DUT. This location is subjected to additional mechanical loads if an LAS is present on the NTB. Due to these constraints, NTB operators use different methods to determine the torque that is applied onto the DUT. These measurements are often not traced to national standards, and, sometimes, this is not even possible. Even if traceability is ensured, the measurement uncertainty for the torque measurement is most likely to be $>0.1 \%$. However, for most NTBs, no uncertainty budget for the torque measurement exists at all.

In September 2015, the 'Torque Measurement in the MN.m Range' project started within the framework of the European Metrology Programme for Innovation and Research (EMPIR). It aims to improve the abovementioned situation by evaluating it and by enabling a torque traceability of up to $5 \mathrm{MN} \cdot \mathrm{m} \mathrm{[3].} \mathrm{This}$ article is based on the preliminary outcomes of the project, and it describes the different methods of torque measurements on NTBs. The traceability and measurement uncertainty of these methods are discussed in order to enable the evaluation of the present measurement uncertainty of NTBs, which is otherwise not possible. Based on this discussion, it is shown that a torque calibration is needed, and recommendations for a calibration procedure on NTBs using a torque transfer standard (TTS) are given to form the basis for traceable torque measurements in the $\mathrm{MN} \cdot \mathrm{m}$ range.

\section{OVERVIEW OF CURRENT TORQUE MEASUREMENT METHODS ON NTBS}

The main objective of torque measurements on NTBs is to determine the torque load to which the DUT is submitted. Therefore, a measurement directly between the LAS and the DUT would be preferable. However, this is not possible for all the different torque measurements, as they do not tolerate additional mechanical loads or need be performed at another location. To account for the special requirements for the different torque measurement methods, alternative locations have been chosen. In Figure 2, an overview of the locations for torque measurements on NTBs is given together with the three most common measurement methods based on [4]. In the following part, the three methods are described, and their location of application is discussed.

Direct torque measurement: A torque transducer consisting of a deformation body and strain gauges is used. This measurement method is feasible at any position of the main shaft of an NTB (see Figure 2).

Torque loads occurring directly at the DUT are in the multi$\mathrm{MN} \cdot \mathrm{m}$ range and, therefore, cannot be traced to any torque standard. Moreover, the influence of the multi-component loading by the LAS cannot be taken into account. A torque measurement between a prime mover and an LAS (without a gearbox) still lacks traceability due to the large torque loads. The

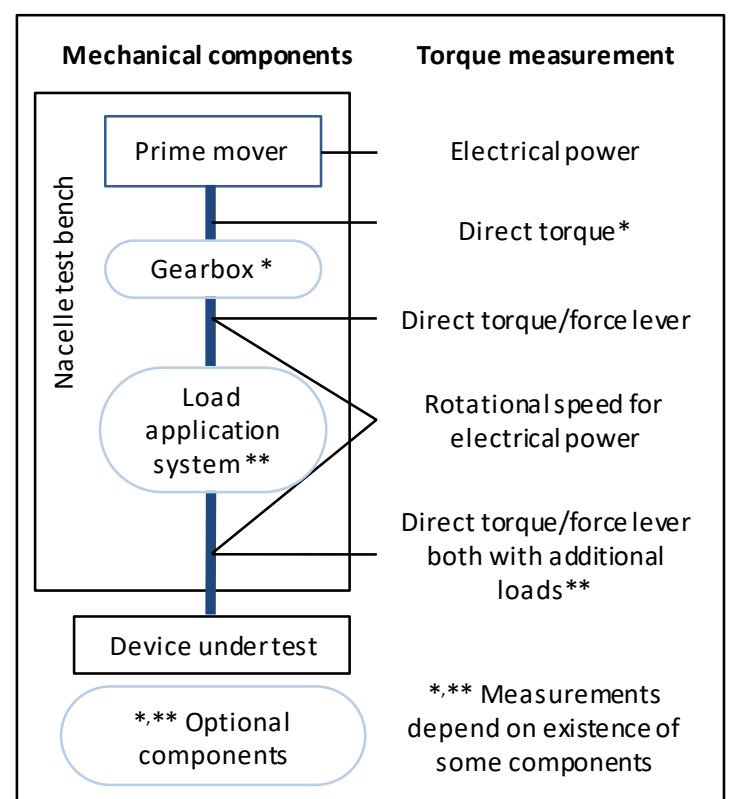

Figure 2. Locations and methods of torque measurements in nacelle test benches

only currently available option for traceable measurements using direct torque measurement is to measure the torque load between the prime mover and the gearbox, as this is much smaller than at the DUT (see Figure 3). If this last option is used, the mechanical efficiency of the gearbox $\eta_{\text {gear }}$ must be considered as an additional influence.

Force lever: A rotating force lever system consists of several force transducers that are connected to the main shaft of the NTB at a certain distance to the centre of rotation. Technically, the same locations as for direct torque measurements are possible for force lever measurements. However, due to the increased measurement uncertainty compared to direct torque measurements, using force lever systems only makes sense at locations where direct torque measurement is not possible, e.g. due to traceability issues for multi-MN $\cdot \mathrm{m}$ measurements. These locations are either between the prime mover and the LAS

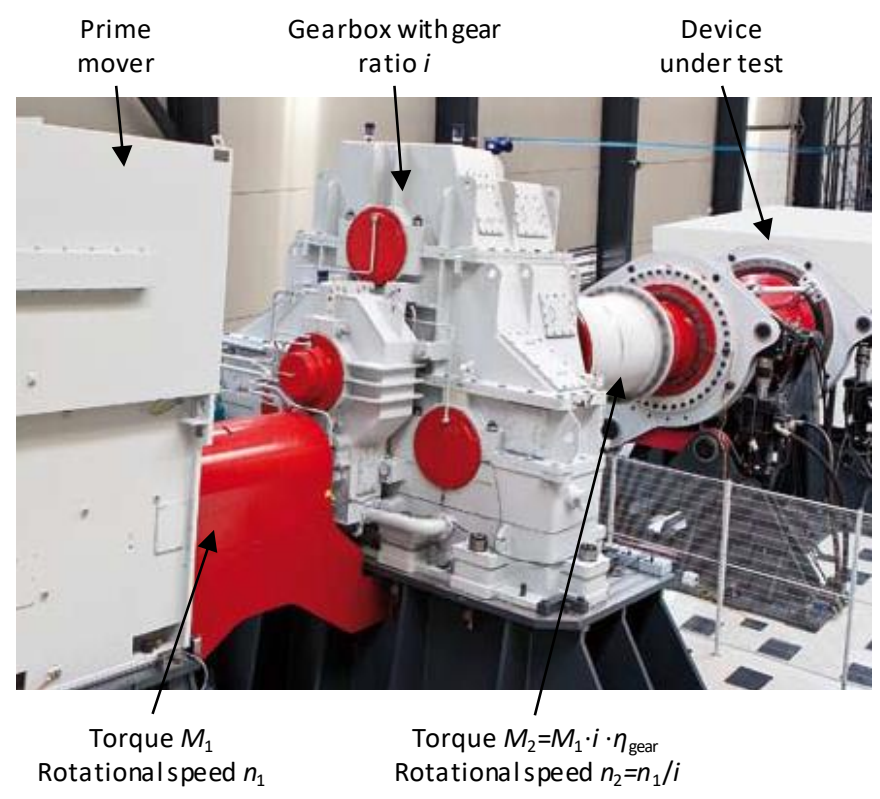

Figure 3. Example of a gearbox in an NTB drive (source: CENER) 
without a gearbox or between the LAS and the DUT. At the second location, the loads from the LAS must be transmitted by the force lever system as well.

Theoretically, a non-rotating force lever measurement at the prime mover in the form of a reaction force measurement would be possible. However, in practice, this is not sensible, as it entails a myriad of other issues from the location of the measurement to the quantification of effects, such as torque bypasses and additional mechanical loads.

Electrical power: Instead of measuring the input torque to the DUT, it is possible to calculate the input power based on the measurement of electrical power. For the calculation of the input torque, the measurement of rotational speed is also needed. The electrical power must be measured as the input to the prime mover. The rotational speed should be measured at the location for which the torque load is calculated: between the LAS and the DUT. This difference between the locations of the two components involved in the torque calculation is the main drawback of this method. It entails influences such as friction and other losses between the place of measurement of the electrical power and the input to the DUT.

Traceability of the abovementioned methods: Not all of the described methods provide complete traceability for the torque measurement. Direct torque measurement is only traceable up to 1.1 $\mathrm{MN} \cdot \mathrm{m}$, which is only sufficient for measurements between the prime mover and the gearbox. All measurements between the LAS and the DUT (direct and force lever measurement) are influenced by the additional mechanical loads, which cannot yet be considered sufficiently. Hence, these methods are not considered in the following section.

\section{TORQUE MEASUREMENT UNCERTAINTY IN NTBS WITHOUT A TORQUE CALIBRATION OF THE SETUP}

This section focuses on the estimation of the uncertainty of torque measurements in an NTB without prior calibration of the entire setup. As the torque measurement methods in NTBs differ greatly, several different influences and their effect on the measurement uncertainty must be accounted for. In addition, some specific influences are found in all NTBs. An overview of the influences on the torque measurement in NTBs is given in Figure 4. In the following sections, the NTB-specific influences are discussed as well as the method-dependent influences. Only traceable torque measurement methods that are not inflicted with a large temporal shift due to their location in respect to the DUT are considered. This leaves two methods: the direct torque measurements between the prime mover and the gearbox with torque loads in the $\mathrm{kN} \cdot \mathrm{m}$ range as well as the force lever measurements between the prime mover and the LAS without large additional loads, as measurements under these conditions would not be traceable.

\subsection{General influences}

Essentially, the measurements in NTBs are under the same influences as those in the reference torque standards using one or several calibrated transducers. However, the effects on the torque measurement are sometimes even larger, and additional contributions to the measurement uncertainty of torque measurement are found in NTBs. In [5], an overview of the measurement uncertainty in reference torque standards is given. This includes the uncertainty based on transducer calibration as well as the ambient conditions, such as temperature and humidity, which are often unstable in NTBs. A correction of the torque measurement based on a temperature and humidity measurement should therefore be made, which requires investigations of the measuring system under different conditions.

Another NTB-specific influence is rotation. A detailed analysis of the effect of rotation on the measurement results can be found in [6]. According to this paper, it can be assumed that the rotation influence for torque transducers in an NTB is mostly negligible, as the rotational speed is fairly small $(<25$ turns per minute). Only for torque transducers between the prime mover and the gearbox can an effect of up to $0.1 \%$ occur, according to [6], due to the much higher rotational speed (400 - 700 turns per minute). An estimation of the effect of rotation on the force lever torque measurement was performed in [7] and was found to be $<0.1 \%$.

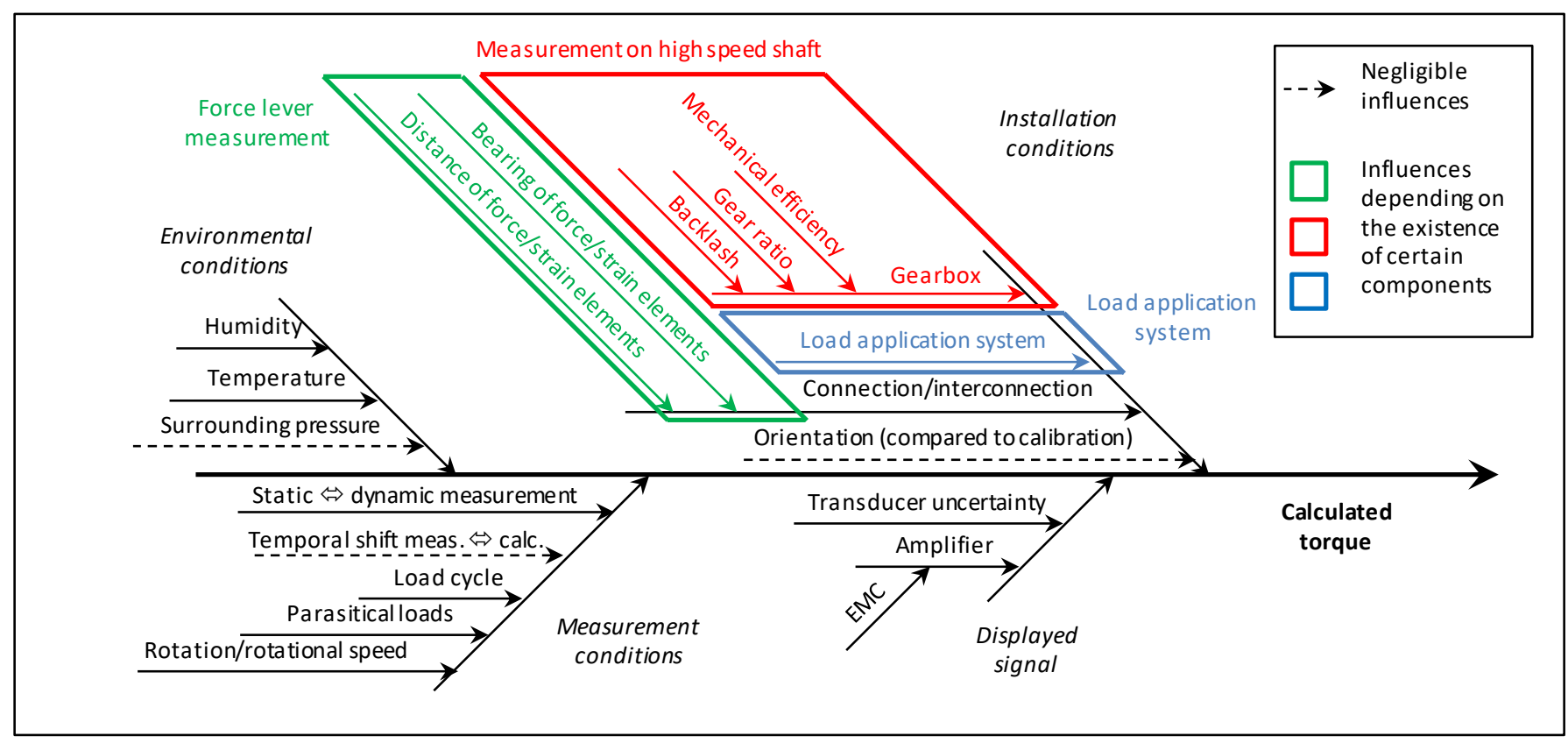

Figure 4. Ishikawa diagram showing the influences of current torque measurement methods on the measurement results in nacelle test benches 
The influence of dynamic mechanical loading compared to a static calibration is extremely difficult to estimate and take into consideration. Scenarios such as an emergency brake lead to impact torque loads that cannot be measured accurately with strain gauge-based transducers. In the case of small acceleration of the drive train or slight changes in the torque load, the effect on the measurement should be relatively small. However, as a dynamic calibration is not possible at the moment, dynamic loading has to be minimised. A measurement uncertainty for the torque measurement can therefore only be given for stationary states in the NTB e.g. only when a constant rotational speed is possible, not for braking scenarios. Especially for the determination of the efficiency of the DUT, only stationary loads are used.

A further influence that can be observed in all NTBs is the load cycle, which differs greatly from the standard calibration cycle and which can have an effect on the torque measurement, e.g. by affecting the hysteresis. In general, this effect is relatively small, but if it is combined with alternating torques (which occur, for example, in brake tests), a larger effect on the torque signal and thus on the measurement uncertainty is expected [8]. The calibration of the transducers should therefore account for this influence.

The influence of parasitical loads and a proposal to consider them are described in [8]. However, this approach is purely empirical, and more research is needed to include this influence into a torque uncertainty budget.

The last general influence is caused by the amplifier. This should be chosen according to the targeted torque measurement uncertainty, meaning a sufficiently precise amplifier has to be employed. However, due to the large number of the electrical components of the NTBs, electromagnetic compatibility (EMC) is an issue for the measurements. Consequently, the EMC effect on the amplifier signal should be checked.

\subsection{Influence of the LAS}

The LAS is responsible for the application of additional static and dynamic wind loads (e.g. thrust, radial forces, and bending moments). There are different construction principles of the LAS regarding the load application and the suspension of the

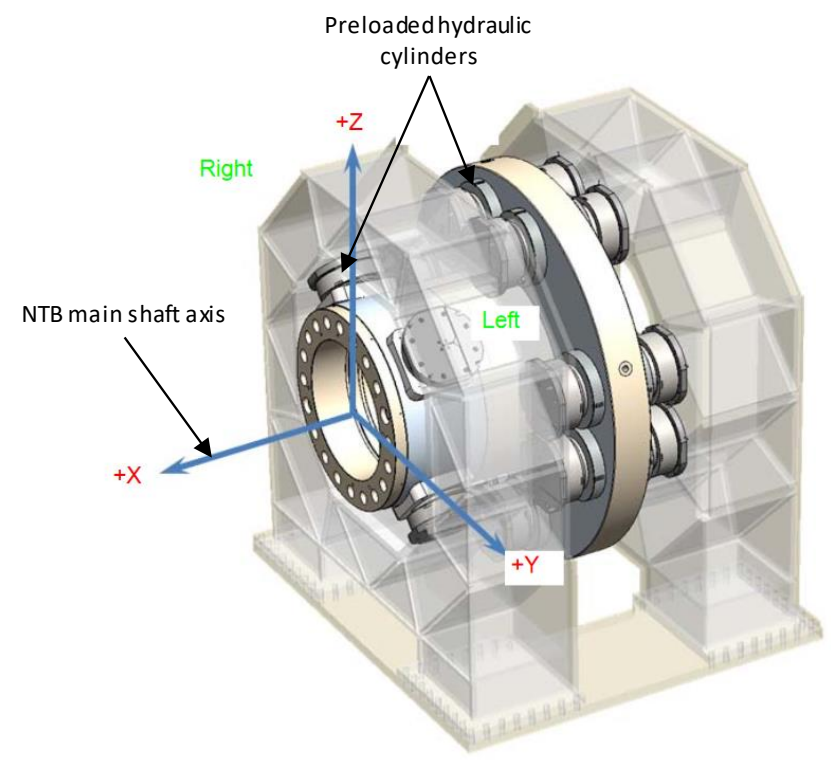

Figure 5. Load application system at CWD Aachen (source: MTS Systems Corporation)
NTB main shaft, e.g. load application by several preloaded hydraulic cylinders (Figure 5) or by a hydraulic hexapod in the form of a Stewart platform (e.g. 10 MW IWES DyNaLab). The suspension of the NTB main shaft can be designed with hydrostatic plain bearings or with roller bearings (e.g. arranged tapered roller bearings). Hydrostatic plain bearings are more expensive, but their use is preferred because of the lower friction torque, lack of stick slip, lower deformation, longer life durability, and high reliability.

Even when no additional loads are applied, the LAS is used for stabilisation, leading to power losses and friction torque during rotation along the main shaft. The calculation of the power loss is crucial for torque measurement between LAS and drive (e.g. electrical input measurement, see Figure 2). This loss is mainly caused by the NTB main shaft suspension and can be accounted for with an efficiency parameter $\eta_{\text {LAS }}$, which depends on the design of the LAS, the rotational speed, and the torque on the drive train as well as the loads applied by the LAS. Because of the abovementioned influences, the traceable measurement of the LAS friction torque under different operation conditions is not possible. However, the efficiency parameter $\eta_{\text {LAS }}$ can be estimated by analytical or numerical methods that are based on fluid dynamics. In the case of suspension with roller bearings, the bearing manufacturer provides analytical calculation methods for the estimation of friction torque. In the case of hydrostatic plain bearings, the calculation methods are based on the descriptions of the Hagen-Poiseuille flow and the corresponding drop of pressure in the plain bearing. For both the suspension methods of the NTB main shaft, the load-dependent parameters - such as the temperature and viscosity of the oil as well as lubricant thickness, local pressure, and the entire system friction coefficient - are estimated. Consequently, the estimation of $\eta_{\text {LAS }}$ requires close consultation between the NTB operator and the LAS manufacturer.

An exemplary estimation of the LAS effect on the torque measurement based on numerical simulation was made in [10]. There it was found that the analysed LAS leads to a friction torque of up to around $1 \mathrm{kN} \cdot \mathrm{m}$ for a $1.5 \mathrm{MN} \cdot \mathrm{m}$ torque load. Assuming an uncertainty of $10-20 \%$ for such a numerical estimation, it is possible to evaluate the overall effect of the LAS on the torque measurement uncertainty.

\subsection{Influence of the gearbox}

To determine the torque load at another part of the drive train, e.g. right at the DUT, the influence of the gearbox has to be considered. For a measurement between the prime mover and the gearbox, this can be expressed in the following equation:

$M=M_{\mathrm{T}} \cdot i \cdot \eta_{\text {gear }}$

with:

$M=$ input torque for DUT

$M_{\mathrm{T}}=$ torque measured at transducer between the prime mover and the gearbox

$i=$ gear ratio

$\eta_{\text {gear }}=$ mechanical efficiency of the gearbox

Gear ratio $i$ is a parameter that is determined by the layout of the gearbox. As this is a fixed constant, apart from small variations due to imprecisions in the manufacturing process, the uncertainty of the gear ratio $i$ is extremely small. This can be neglected if the measurement values are averaged over an integer number of rotations of the shaft where the measurement is taken and, if possible, even over an integer number of rotations of the 
shaft for which the torque load is calculated. Ideally, several full rotations of the gearbox are used for the measurement.

The mechanical efficiency of the gearbox $\eta_{\text {gear }}$ is dependent on the load (torque and rotational speed) as well as on the condition of the gearbox (e.g. oil condition and temperature). Therefore, precise information on $\eta_{\text {gear }}$ for the entire load and condition range of the NTB is necessary. Ideally, the measurement uncertainty is provided, too. This is, however, mostly not the case, and an uncertainty for $\eta_{\text {gear }}$ must be assumed based on experience. If a transfer torque transducer for the $\mathrm{MN} \cdot \mathrm{m}$ range is available, this assumption can be verified.

An example of the measurement uncertainty of a torque measurement caused only by the presence of a gearbox is given in the following section.

The assumed input is based on the general information known about NTBs and gearboxes. $i$ is considered to be a constant factor without a measurement uncertainty:

$$
\begin{aligned}
M & =4750 \mathrm{kN} \cdot \mathrm{m} \\
M_{\mathrm{T}} & =100 \mathrm{kN} \cdot \mathrm{m} \\
i & =50 \\
\eta_{\text {gear }} & =0.95
\end{aligned}
$$

measurement uncertainty of torque transducer:

$u_{\mathrm{MT}}=0.1 \mathrm{kN} \cdot \mathrm{m}$ (normal distribution)

measurement uncertainty of gearbox efficiency:

$u_{\eta}=0.02 / \sqrt{3}$ (rectangular distribution)

The standard measurement uncertainty of the calculated torque is:

$u_{\mathrm{M}}=\sqrt{u_{\mathrm{MT}}^{2} \cdot c_{\mathrm{MT}}^{2}+u_{\eta}^{2} \cdot c_{\eta}^{2}}$

The sensitivity coefficients for the torque transducer and the gearbox efficiency are:

$$
\begin{array}{r}
c_{\mathrm{MT}}=i \cdot \eta_{\text {gear }} \\
c_{\eta}=M_{\mathrm{T}} \cdot i \\
u_{\mathrm{M}}=\sqrt{\begin{array}{l}
(4.75 \mathrm{kN} \cdot \mathrm{m})^{2} \\
+(57.73 \mathrm{kN} \cdot \mathrm{m})^{2}
\end{array}}=58 \mathrm{kN} \cdot \mathrm{m}
\end{array}
$$

The expanded relative measurement uncertainty of the calculated torque is:

$W_{\mathrm{M}}(k=2)=2 \cdot \frac{58 \mathrm{kN} \cdot \mathrm{m}}{4750 \mathrm{kN} \cdot \mathrm{m}}=2.4 \%$

The standard uncertainty of the mechanical efficiency $\eta_{\text {gear }}$ cannot be determined correctly, so the result has to be used with care. However, the effect of the uncertainty of the mechanical

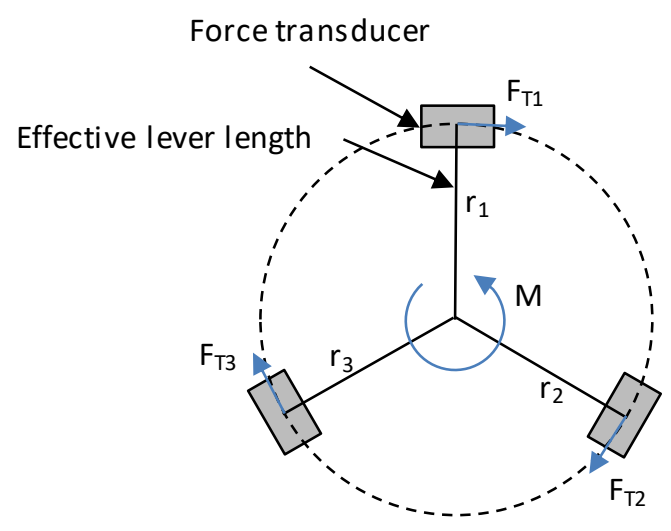

Figure 6. Sketch and parameter definition for a force lever system. efficiency $\eta_{\text {gear }}$ on the overall result is clarified, as it constitutes the main part of the overall torque measurement uncertainty.

\subsection{Influence of force lever measurement}

One way of avoiding the problem of the traceability of direct torque measurement in the $\mathrm{MN} \cdot \mathrm{m}$ range is to employ a torque transducer consisting of force transducers at a fixed distance to the main centre of rotation. The number of force transducers is variable. A definition sketch for the setup of a force lever system and its parameters is shown in Figure 6. Sketch and parameter definition for a force lever system.. Equation (5) presents the calculation of the resulting torque assuming equally distanced transducers.

$$
\begin{aligned}
& M=\sum_{i=1}^{n}\left(F_{\mathrm{T}, i} \cdot \mathrm{r}_{\mathrm{i}} \cdot \eta_{\mathrm{con}}\right) \\
& \text { with: } \\
& M=\text { input torque for DUT } \\
& F_{\mathrm{T}, i}=\text { force measured at transducers } \\
& r_{i}=\text { distance of force measurement from the main axis } \\
& n=\text { number of force transducers used for the } \\
& \text { measurement } \\
& \eta_{\mathrm{con}}=\text { mechanical efficiency of the connection between } \\
& \text { the force transducers and the main shaft }
\end{aligned}
$$

The measurement uncertainty of the force measurement can be based on a calibration. However, the influence of parasitic loads on the force transducer due to alignment errors and deformation under load cannot be sufficiently accounted for. Some recommendations and a mathematical model for determining the additional measurement uncertainty for force measurements in the $\mathrm{MN}$ range were given in the EMRP project called 'Force traceability in the meganewton range' [11]. The influence of the slightly dynamic loading of the force transducers cannot be taken into account at all.

The effect of the uncertainty of the distance $r$ on the overall torque measurement uncertainty is very difficult to estimate, as $r$ changes under load. This change $\Delta r$ is load-dependent, making it a systematic effect that cannot be measured. Moreover, the value of $r$ only decreases under pure torque load if the force transducers are under a tension load. As a correction of this effect is not possible without detailed measurements of $r$ under load, $\Delta r$ must be estimated and taken as a direct contribution to the overall measurement uncertainty of $M$ according to [12], [13]. $\Delta r$ is much bigger than the uncertainty of $r$ itself, which can be obtained by a calibration, and it is also bigger than its own uncertainty.

The influence of the connection between the force transducers and the main shaft can be accounted for using a factor $\eta_{\text {con }}$. This factor can technically be treated similarly to $\eta_{\text {gear }}$. However, the effect on the force lever system torque measurement is expected to be much smaller as the effect is only indirect. Moreover, $\Delta r$ has a much larger influence. Therefore, $\eta_{\text {con }}$ can be considered to be negligible as long as $\Delta r$ cannot be quantified satisfactorily.

In the following, an example of a calculation of the measurement uncertainty contribution caused by a torque measurement using a force lever system is presented. Similar to the example of the influence of a gearbox given above, the values given are based on estimations:

$$
\begin{aligned}
& M=4800 \mathrm{kN} \cdot \mathrm{m} \\
& n=3 \\
& F_{\mathrm{T} 1}=F_{\mathrm{T} 2}=F_{\mathrm{T} 3}=1600 \mathrm{kN} \\
& r_{1}=r_{2}=r_{3}=1 \mathrm{~m}
\end{aligned}
$$


$\eta_{\text {con }}=1$ (neglected in the example)

measurement uncertainty of distance $r$.

$u_{\mathrm{r}} \quad=0$ (neglected in the example)

measurement uncertainty of force transducers:

$u_{\mathrm{FT}}=1 \mathrm{kN}$ (normal distribution)

$\Delta r=0.005 \mathrm{~m}$ (systematic error)

The standard measurement uncertainty of the calculated torque is:

$u_{\mathrm{M}}=\sqrt{3 \cdot u_{\mathrm{FT}}^{2} \cdot c_{\mathrm{FT}}^{2}+3 \cdot \Delta r^{2} \cdot c_{\mathrm{r}}^{2}}$

The sensitivity coefficients for force transducers and the distance from the centre of rotation are:

$$
\begin{array}{r}
c_{\mathrm{FT}}=r \\
c_{\mathrm{r}}=F_{\mathrm{T}} \\
u_{\mathrm{M}}=\sqrt{\begin{array}{l}
3 \cdot(1 \mathrm{kN} \cdot \mathrm{m})^{2} \\
+3 \cdot(8 \mathrm{kN} \cdot \mathrm{m})^{2}
\end{array}=14 \mathrm{kN} \cdot \mathrm{m}}
\end{array}
$$

The expanded relative measurement uncertainty of the calculated torque is:

$W_{\mathrm{M}}(k=2)=2 \cdot \frac{14 \mathrm{kN} \cdot \mathrm{m}}{4800 \mathrm{kN} \cdot \mathrm{m}}=0.6 \%$

The influence of the change of distance $r$ under load might have been overestimated in the example; however, the significance of the influence is clearly shown. For the overall torque measurement uncertainty, the uncertainties of $r$ itself and of $\Delta r$ are not considered, as they should be comparatively small. Even without these components, the expanded relative uncertainty of $M$ results in $W_{\mathrm{M}}(k=2)=0.6 \%$.

In further studies within the same project framework, several force lever systems have been designed and analysed (see [7] and [14]). Additional influences on the measurement that have been found in these studies are the additional mechanical loads and the temperature. These influences should be considered with care when using a force lever system in an NTB. Especially for the additional mechanical loads, a specific design of the force lever system to transmit the load through the force lever system has to be chosen (e.g. roller bearing, as in [7]).

\subsection{Summary of influences on torque measurement and their effect on measurement uncertainty}

Most of the influences shown in Figure 4. Ishikawa diagram showing the influences of current torque measurement methods on the measurement results in nacelle test benches and their effect on the uncertainty for torque measurements on NTBs can be estimated. The uncertainty is larger than the demanded $0.1 \%$, due to a variety of influences on the measurement. Moreover, influences derived from an LAS, such as friction and additional loads, are still not accounted for, and a verification of the estimation is not possible. In summary, it can be stated that a calibration of the torque measurement on NTBs is necessary to obtain better precision of the torque measurements through improving the estimation of the measurement uncertainty, thus also improving the efficiency determination of nacelles. For the torque calibration of an NTB, a TTS in the $\mathrm{MN} \cdot \mathrm{m}$ range is required. Moreover, a calibration procedure considering all occurring influences on NTBs is necessary. In the subsequent section, recommendations for such a procedure are summarised.

\section{DEVELOPMENT OF A TORQUE CALIBRATION PROCEDURE FOR NACELLE TEST BENCHES}

Based on the descriptions and discussions in previous sections, recommendations for a torque calibration procedure of NTBs are given in the following subsections. The calibration recommendations are based on EURAMET cg-14 [15] and ISO 7500-1 [16]. The recommended procedure focuses on torque calibration without any additional mechanical loads but also includes a section on further tests to analyse the multicomponent effects on the torque measurement.

\subsection{Requirements for a torque transfer standard}

As was shown in the previous sections, it is possible to measure MN.m torque loads in NTBs; however, the measurement uncertainty is not small enough for this purpose. Therefore, it is inevitable that a TTS should be used to calibrate the NTB. This transducer must be characterised over the torque range of the NTB. This was not possible before the start of the project 'Torque measurement in the $\mathrm{MN} \cdot \mathrm{m}$ range', but it was found to be a requirement for the improvement of the torque measurement in NTBs. Within the project, two options of traceability with TTS have been developed: extrapolation and force lever systems. These can be used for the purpose of calibrating the torque signal of an NTB.

It is also advantageous to have knowledge of the behaviour under temperature (and humidity) of the TTS, as the NTB calibration cannot be performed under laboratory conditions. Furthermore, the transducer should withstand the occasionally occurring additional mechanical loads in the NTB, as it has to be installed directly in front of the DUT. The general requirements for the TTS are its weight, size, and adaptability to the NTB, which has to be checked for each TTS and NTB combination individually.

\subsection{General measurement conditions}

The TTS has to be placed directly between the NTB and the DUT (see Figure 2. Locations and methods of torque measurements in nacelle test benches) for the torque calibration measurements because the influences of an existing gear box or LAS (see Sections 3.2 and 3.3) would otherwise have to be estimated. The alignment of the TTS on the NTB has to be very precise, as misalignments can lead to additional permanent loads, such as axial force, lateral forces, or bending moments.

Furthermore, a same-time measurement of the NTB torque and TTS signal is necessary either over one shared data acquisition system or two synchronised ones. Moreover, the rotational speed has to be recorded in order to determine any influences due to rotation and to perform a calibration with calibration maps (see section 4.6).

The torque signals (NTB and T'TS) and the rotational speed have to be recorded in an adequate frequency, taking into account the rotational speed of the test bench and the envisioned resolution of the encoder or rotation speed signal. All results should be averaged for each load step for at least one turn of the main shaft. An averaging over a larger number of turns results in the filtration of mechanical noise but also makes time-dependent effects like creeping or an instable test bench control invisible. Good knowledge of the NTB behaviour is, therefore, essential for determining the precise number of turns.

As the recorded (non-averaged) data can be used to determine any temporal phase shifts between the measurements, it should be stored in order to also analyse the directly measured signals for the entire testing time. 
a) Static zero signal

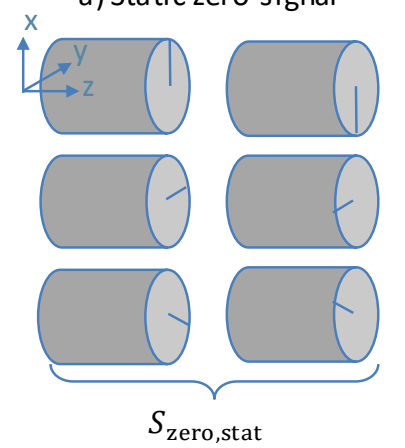

b) Rotational zero signal

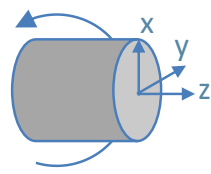

$S_{\text {zero,rot }}$

Figure 7. Explanatory sketch of the procedure for zero signal determination a) static zero and b) rotational zero.

It should be ensured that the measurement and recording of humidity and temperature values are as close to the TTS and the NTB-torque measurement as possible during the measurement. Ideally, the temperature gradient on the TTS should be measured. All transducer signals should be corrected for changes in the environmental conditions (see [5]). To ensure relatively stable environmental conditions, the NTB should be in operation with the entire setup prior to the calibration.

As the calibration of an NTB is not possible without a DUT, a suitable test object should be available for the calibration. Ideally, a DUT with full control access is used for the calibration, as several scenarios depend on the controlled change of electrical braking power asserted by the generator. An alternative to using an entire nacelle for the calibration is using only a generator. This often provides the necessary access to the control but comes with the disadvantage of not being able to perform full multicomponent measurements, as the generator is not built for this purpose.

\subsection{Preparatory tests}

As there are always high-powered electrical machines in multiMW NTBs, the EMC of the TTS should be tested by purposely causing possible interference e.g. a test with a TTS in an uninstalled situation prior to installation. Furthermore, the EMC for the NTB-torque measurement should be ensured.

It is also advised that some general test with a range of torque loads and rotational speed in advance of the torque calibration should be undertaken to optimise the control system, as the additional TTS leads to a change in the mechanical behaviour of the entire setup. Furthermore, the influence of the warming up of the NTB can be studied during this time if no reliable knowledge of the behaviour is available.

\subsection{Determination of the zero signal}

A crucial step in the calibration of the NTB torque is the determination of the zero signal. The quality and correctness of the signal affect all other results during the calibration. There are two main issues in determining the zero signal. Firstly, the TTS cannot be dismounted after each measurement cycle; therefore, the zero signal has to be established with the entire setup. Secondly, the transducer is loaded by a bending moment and a lateral force due to its own weight, which is different to the situation under rotation, where the averaging of the signal of an integer number of turns leads to the deletion of this effect. Two options are suggested to account for these issues: static and rotational zero point determination. Ideally, both are performed for comparison purposes.

\subsubsection{Static zero signal}

All zero signals of the NTB torque measuring device and the TTS have to be determined as an average over differently rotated positions with respect to the measurement axis. The rotation should be performed for the entire main shaft of the NTB without the mounting and dismounting of the TTS. Depending on the measurement method, $6.60^{\circ}$ rotations (force lever system) or $3.120^{\circ}$ rotations (direct torque measurement) are suitable. This option is visualised in Figure 7(a). The static zero signal can be calculated using the following equations:

$$
\begin{array}{ll}
S_{\text {zero.stat }} & =1 / p \cdot \sum_{i=1}^{p} \bar{S}_{i} \\
\text { with } \quad \bar{S}_{i}=1 / n \cdot \sum_{j=1}^{n} S_{j} \\
\text { and } \quad n=t_{\text {meas }} \cdot f_{\text {sample }}
\end{array}
$$

where $p$ is the number of measurement points, $t_{\text {meas }}$ is the duration of each measurement, and $f_{\text {sample }}$ is the sampling frequency of the measurement.

\subsubsection{Rotational zero signal}

A rotational zero-point determination can be made either before connecting the TTS to the DUT or with the entire setup. In both cases, the signal is recorded over an integer number of turns and averaged in the postprocessing (see Figure 7(b)). The disconnected option has the advantage that no friction torques occur, but it has the disadvantages of the transducer needing to be disconnected, which cannot be ensured between calibration sequences, and of the installation condition not being exactly the same as the condition during the calibration. The connected option leads to an additional friction torque but can easily be performed between tests.

In [17], it was found that the friction torque in the connected zero-signal procedure can be minimised by employing the DUT generator as a second engine to compensate for the losses in the drive train. For this control mode, full access to the NTB control is required.

The rotational zero signal can be calculated using the following equations:

$$
\begin{aligned}
& S_{\text {zero,rot }}=1 / m \cdot \sum_{i=1}^{m} S_{i} \\
& \text { with } \quad m=n_{\min } \cdot k \cdot f_{\text {sample }}
\end{aligned}
$$

where $n_{\min }$ is the minimum speed of rotation, $k$ is the number of turns of the drive train, and $f_{\text {sample }}$ is the sampling frequency of the measurement

\subsection{Quasi-static torque calibration}

To achieve traceability to the TTS calibration and for a comparison with the static calibration of the TTS, it is advised that a quasi-static calibration of the NTB should be performed first. This is a normal torque calibration according to [15], under a constant minimum rotational speed. Instead of using three different installation positions as requested by [15], three repetitions without dismounting the TTS are sufficient. The repeated installation of the TTS would be helpful but is not necessary, as the TTS is rotated during the calibration, and the control system applies additional lateral forces and bending moments. 


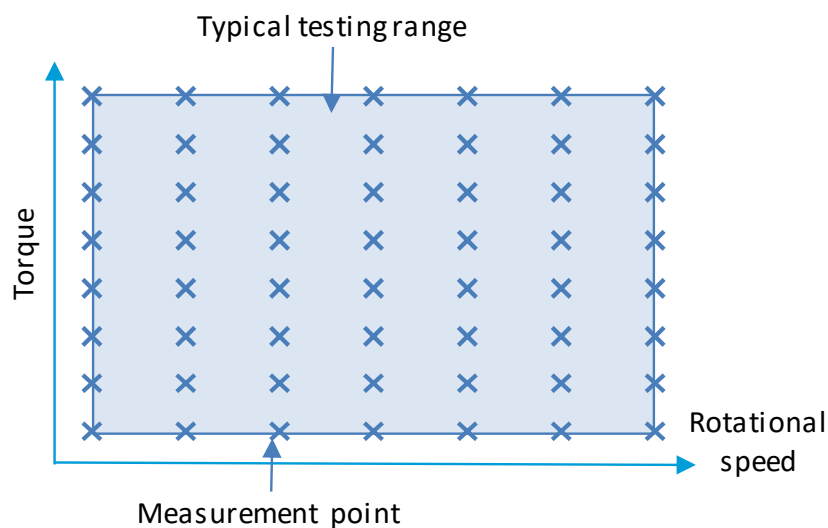

Figure 8. Schematic drawing of a calibration map based on the typical testing range of an NTB and with a uniform distribution of the measurement points.

\subsection{Calibration maps}

Identifying the influence of the rotational speed on the torque measurement is an important part of the calibration of an NTB, as most NTBs cannot apply torque loads without rotating. A simple solution to account for this influence in the NTB calibration is the use of calibration maps. This is a combination of all possible torque and rotational speed loads. A similar procedure has been suggested for rotary power measurements [18]. In the first step, the typical testing range (rotational speed and torque values) of the NTB has to be determined. This is represented by the blue rectangle in Figure 8 . The calibration should then cover the entire range by repeating the standard calibration with different combinations of torque load and rotational speed, as shown by the crosses in Figure 8.

If this procedure is not possible due to insufficient access to the control values, the calibration must be performed in the median of the typical testing range requesting a standard DUT. Load steps for the torque calibration should be based on the typical maximum torque loads of the NTB for different DUTs. Several standard calibrations, one for each typical load, should be performed. The increments of the load steps should also represent the typical NTB situation; e.g. if a torque load of $4-$ $5 \mathrm{MN} \cdot \mathrm{m}$ is typical, more load steps in this range and fewer below $4 \mathrm{MN} \cdot \mathrm{m}$ are required. It is reasonable to omit rotational speed steps that are near to an eigenfrequency of the system and would lead to dynamic effects on the torque signal.

During the performance of one calibration map test, each measurement point should preferably be reached twice to evaluate the hysteresis. The order of the measurement points within the calibration map must be defined with care, as control influences might lead to different results depending on the order. In Figure 9, two examples of the resulting load-step sequences are given, where the torque load is the fixed parameter, and the rotational speed is altered on each torque step. It is also possible to fix the rotational speed and alter the torque load on each speed step. Thus, several different measurement sequences based on the same basic calibration map are possible. Generally, the typical load sequences of the NTB under normal conditions should be used to decide for a load sequence.

Each load step has to be held for the time that the NTB needs to stabilise its control plus a minimum of six rotations. A longer measurement time would also give an indication of the stability of the NTB control. A repetition of the calibration map sequence

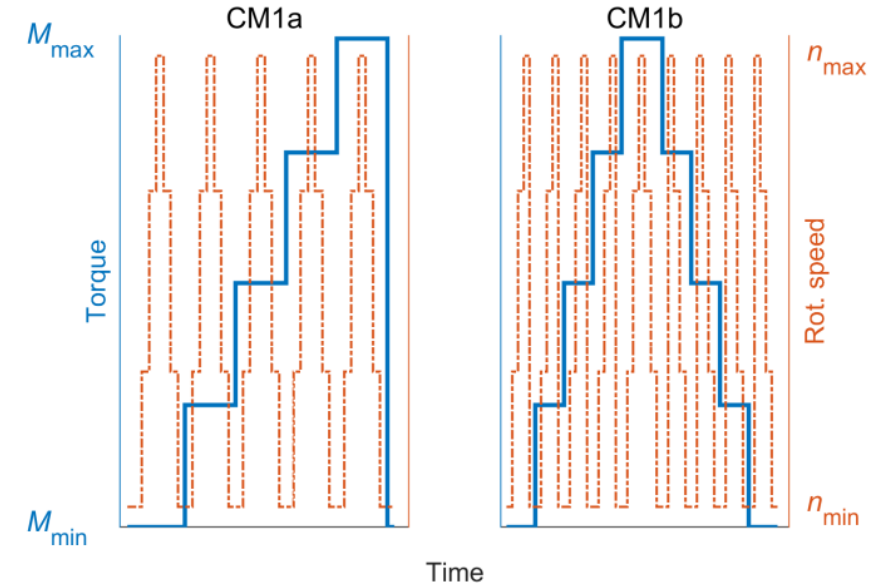

Figure 9. Example of two different load-step sequences for a calibration with torque being increased (and decreased) in one (two) staircase(s) and increasing and decreasing rotational speeds on each torque step.

of at least three times is recommended to determine the repeatability of the NTB.

\subsection{Multi-component effects on the torque measurement}

It is expected that additional mechanical loads of the NTB cannot be ignored. A deviation into purposely applied loads and loads due to control issues has to be made. It is assumed that the occurrence of LAS-induced loads due to control issues is random if the setup is operated in pure torque mode for the calibration. Their effect is therefore accounted for in the torque measurement uncertainty within the part of repeatability.

If the aim of the calibration is an additional calibration of the NTB torque under larger multi-component loads, which are applied on purpose, and a similar procedure for this calibration as for the effect of rotational speed is proposed. Examples for torque and axial force as well as for torque and axial and lateral force are given in Figure 10.

\subsection{Additional tests}

If the NTB is used for a wide range of DUTs, it would be sensible to perform calibrations in the full torque range of the NTB and in partial ranges. Ideally, different DUTs are used for the different ranges.

Most NTBs have one main direction of rotation. Therefore, only one direction of torque loading is needed. However, the

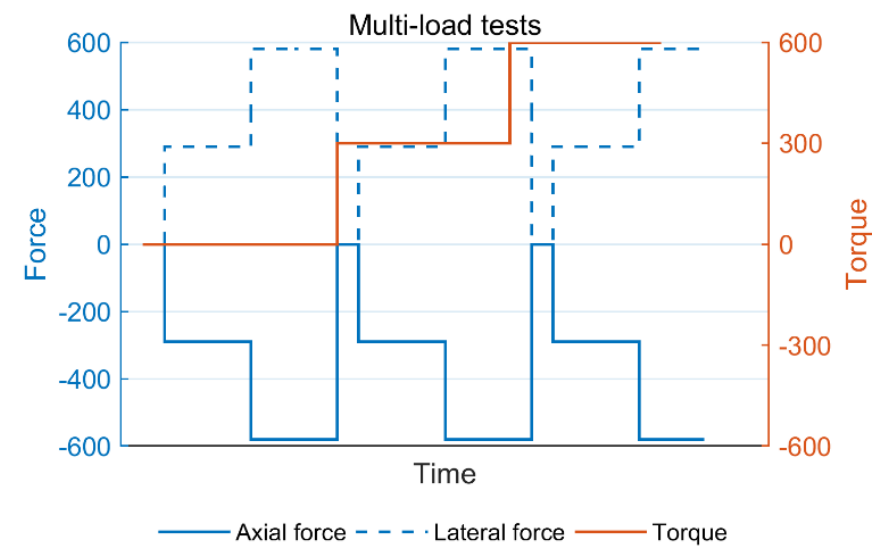

Figure 10. Example of a load sequence for the combination of lateral force, axial force, and torque load. 
influence of possible zero-crossings on the torque measurement system of the NTB should be tested, e.g., by torque loads in the opposite direction. The zero-return and the hysteresis can thus be determined. Furthermore, the influence of braking scenarios on the torque measurement should be tested and analysed. These tests should not be included in the calculation of a torque measurement uncertainty as long as they are not required in a typical loading scenario that is in need of high-precision torque measurement.

Continuous measurement to determine creeping is suggested. This can be performed by recording the torque signals after the end of a calibration map for several minutes.

\subsection{Evaluation}

As already mentioned on several occasions in this section, it is important to use averaged torque values over an integer number of rotations for the evaluation. A constant number should be chosen for all evaluations.

It is almost impossible to predict all resonance frequencies of such a complex experimental setup in advance; therefore, the resulting data has to be checked for possible dynamic influences. This data should best be deleted from the evaluation.

No class definition should be made. An evaluation of all the determined components, indication deviation, and measurement uncertainty is necessary.

\section{CONCLUSIONS}

This paper gives an overview of torque measurement methods in NTBs and specific influences on the torque measurement uncertainty. It was found that the current measurement methods do not fulfil the envisioned measurement uncertainty of NTB operators. Therefore, a torque calibration with a TTS is inevitable. In the final section, a detailed description of recommendations for performing and evaluating such a calibration are given based on existing calibration guidelines.

The project 'Torque Measurement in the MN.m Range' addressed several of the issues that were discussed in this article. The main outcome is the test of a torque calibration of an NTB. A $5 \mathrm{MN} \cdot \mathrm{m}$ TTS with multi-component capabilities was calibrated up to $1.1 \mathrm{MN} \cdot \mathrm{m}$ [19], and an extrapolation to $5 \mathrm{MN} \cdot \mathrm{m}$ was applied [20]. This TTS was then installed in the 4 MW NTB at the CWD Aachen, Germany. Results and a detailed description of the measurement campaign can be found in [17] and [21].

The torque measurement uncertainty of force lever systems has been further investigated within the project, and recommendations for the design and determination of a measurement uncertainty are given. Several publications on this topic have been made ([7], [14], [22]) and are being made ([23][25]) to develop a design that fulfils the requirements of NTB operators.

\section{ACKNOWLEGEMENTS}

The EMPIR initiative is co-funded by the European Union's Horizon 2020 research and innovation programme and the EMPIR Participating States. The discussions and input of all the partners in the project as well as of their colleagues are greatly appreciated.

This paper is an extended version of a conference contribution for the 2017 TC3 IMEKO conference in Helsinki, Finland [1]

\section{REFERENCES}

[1] S. Kock, G. Jacobs, D. Bosse, P. Weidinger, Torque measurement uncertainty in multi-MW nacelle test benches, Proc. of the Conference for Wind Power Drives, Aachen, Germany, 7-8 June 2017.

[2] D. Peschel, D. Mauersberger, D. Schwind, U. Kolwinski, The new 1.1 $\mathrm{MN} \cdot \mathrm{m}$ torque standard machine of the PTB Braunschweig / Germany, Proc. of the 19 $9^{\text {th }}$ Conference on "Force, Mass and Torque Measurement", Cairo, Egypt, 19 - 23 February 2005. Online [Accessed 16 September 2019]:

https://www.imeko.org/publications/tc3-2005/IMEKO-TC32005-KL-001u.pdf

[3] EMPIR project "Torque Measurement in the MN·m Range". Online [Accessed 16 September 2019]:

https://www.ptb.de/emrp/torquemetrology.html

[4] S. Kock, D. Bosse, N. Eich, G. Foyer, N. Medina, J. M. Quintanilla Crespo, L. Vavrecka, J. Ala-Hiiro, Report on existing nacelle test benches and their boundary conditions, Deliverable in EMPIR 14IND14 Project [unpublished] 2016.

[5] D. Röske, Messunsicherheiten bei der Drehmomentmessung mit Referenzmesseinrichtungen, Tech. Mess. 78, 2 (2011) pp. 77-87 [in German].

[6] A. Brüge, Influence of rotation on rotary torque transducers calibrated without rotation, Proc. of the XIV IMEKO World Congress, Tampere, Finland, 2-6 June 1997.

[7] S. Kock, G. Jacobs, D. Bosse, J. Gnauert, Conception of $5 \mathrm{MNm}$ torque transducer for wind turbine test benches, Conference " 5 . Tagung Innovation Messtechnik", Vienna, Austria, 11 May 2017.

[8] D. Röske, D. Peschel, Investigations into the alternating torque calibration of torque transducers, Proc. of the XIV IMEKO World Congress, Tampere, Finland, 2-6 June 1997.

[9] G. Wegener, J. Andrae, Measurement uncertainty of torque measurements with rotating torque transducers in power test stands, Proc. of the $19^{\text {th }}$ Conference on "Force, Mass and Torque Measurement", Cairo, Egypt, 19 - 23 February 2005. Online [Accessed 16 September 2019]:

https://www.imeko.org/publications/tc3-2005/IMEKO-TC32005-049u.pdf

[10] S. Kock, G. Jacobs, D. Bosse, G. Foyer, Influences on MN·m torque measurement in multi-MW nacelle test benches, Proc. of the DEWEK 2017 13 $3^{\text {th }}$ German Wind Energy Conference, Bremen, Germany, 17-18 October 2017, Poster.

[11] EMRP project: "Force traceability within the meganewton range - WP4 Improved traceability". Online [Accessed 13 October 2016]: http://www.ptb.de/emrp/2387.html

[12] I. H. Lira, W. Wöger, The evaluation of standard uncertainty in the presence of limited resolution of indicating devices, 1997 Meas. Sci. Technol. 8441.

[13] JCGM 100:2008: Joint Committee for Guides in Metrology, Evaluation of measurement data - Guide to the expression of uncertainty in measurement, 2008.

[14] R. M. Lorente, N. Medina, M. A. Sáenz, M. Á. Sebastián, Torque traceability for nacelle's test benches: a design proposal, Proc. of the 23rd IMEKO TC3, Helsinki, Finland, 30 May - 01 June 2017. Online [Accessed 16 September 2019]: https://www.imeko.org/publications/tc3-2017/IMEKO-TC32017-004.pdf

[15] Euramet e.V., Guidelines on the Calibration of Static Torque Measuring Devices, 2011.

[16] Beuth, DIN EN ISO 7500-1 Metallic materials - Calibration and verification of static uniaxial testing machines - Part 1: Tension/compression testing machines - Calibration and verification of the force-measuring system.

[17] P. Weidinger, G. Foyer, S. Kock, J. Gnauert, R. Kumme, Development of a torque calibration procedure under rotation for nacelle test benches, Conference "The Science of Making Torque from Wind", Milano Italy, 20-22 June 2018.

[18] A. Brüge, H. Pfeiffer, A standard for rotatory power measurement, Acta IMEKO 8 (2019) 3, pp. 48-58. 
[19] C. Schlegel, H. Kahmann, R. Kumme, MN · m torque calibration for nacelle test benches using transfer standards, Acta IMEKO 5 (2016) 4, pp. 12-18. Online [Accessed 16 September 2019]: http://dx.doi.org/10.21014/acta imeko.v5i4.414

[20] P. Weidinger, G. Foyer, J. Ala-Hiiro, C. Schlegel, R. Kumme, Investigations towards extrapolation approaches for torque transducer characteristics, Proc. of the XXII IMEKO World Congress, Belfast, United Kingdom, 3-6 September 2018. Online [Accessed 16 September 2019]: http://dx.doi.org/10.1088/1742-6596/1065/4/042057

[21] P. Weidinger, G. Foyer, S. Kock, J. Gnauert, R. Kumme, Procedure for torque calibration under constant rotation investigated on a nacelle test bench, Conference "Sensoren und Messsysteme", Nuremberg, Germany, 26-27 June 2018.

[22] G. Foyer, H. Kahmann, Design of a force lever system to allow traceable calibration of $\mathrm{MN} \cdot \mathrm{m}$ torque in nacelle test benches, Conference "Sensoren und Messsysteme", Nuremberg, Germany, 26-27 June 2018.

[23] R. M. Lorente, N. Medina, M. A. Sáenz-Nuño, M. A. SebastiánPérez, Study of influences in CEM's new transfer standard for torque measurements in the MNm range, Proc. of the XXI
IMEKO World Congress, Belfast, United Kingdom, 3-6 September 2018. Online [Accessed 16 September 2019]: http://dx.doi.org/doi:10.1088/1742-6596/1065/4/042039

[24] J. Gnauert, G. Jacobs, S. Kock, D. Bosse, Measurement uncertainty estimation of novel torque transducer for wind turbine test benches, Proc. of the XXII IMEKO World Congress, Belfast, United Kingdom, 3-6 September 2018. Online [Accessed 16 September 2019]:

http://dx.doi.org/10.1088/1742-6596/1065/4/042050

[25] G. Foyer, H. Kahmann, A finite element analysis of effects on force lever systems under nacelle test bench conditions, Proc. of the XXII IMEKO World Congress, Belfast, United Kingdom, 3-6 September 2018. Online [Accessed 16 September 2019]: http://dx.doi.org/10.1088/1742-6596/1065/4/042006

[26] G. Foyer, S. Kock, Measurement uncertainty evaluation of torque measurements in nacelle test benches, Proc. of the $23^{\text {rd }}$ IMEKO TC3 Conference, Helsinki, Finland, 30 May - 1 June 2017. Online [Accessed 16 September 2019]: https://www.imeko.org/publications/tc3-2017/IMEKO-TC32017-010.pdf 